\title{
Estudio exploratorio en línea sobre las opiniones de los padres respecto de la donación de órganos: de las decisiones individuales a las conversaciones en familia
} An online explorative study towards parents' opinions
about organ donation: from individual decisions to family
discussions

\author{
Dra. Marion J. Siebelink ${ }^{a}$, Dr. Marcel J.I.J. Albers ${ }^{b}$, Prof. Petrie F. Roodbol ${ }^{c}$ y \\ Prof. Harry B.M. van de Wield
}

\begin{abstract}
Resumen
Introducción. Muchos factores influyen sobrelas decisiones de los padres acerca de la donación de órganos de sus hijos. Uno de tantos factores puedeser una conversación previa en un contexto no crítico. La pregunta que surge es si los padres han considerado este tema con respecto a ellos mismos y si han hablado del tema entre sí o con sus hijos.

Métodos. Encuesta exploratoria inicial realizada a través de Internet para investigar estas charlas, las opiniones de los padres sobre brindar clases en la escuela acerca de la donación, el umbral de edad para que un niño decida respecto de la donación y los factores determinantes que influyen en estos aspectos. Se envió el cuestionario a 14000 miembros del panel. Se seleccionó a los encuestados que tenían hijos menores de 16 años.

Resultados. La tasa de respuesta del cuestionario fue del 35\%. De los 1146 encuestados con hijos, el $84 \%$ habían conversado sobre este tema con su pareja y, en promedio, el $46,5 \%$ habían conversado con sus hijos de 10 a 16 años de edad. No se halló una relación con la religión y el nivel educativo de los padres. El 69\% de los padres tuvieron una opinión positiva acerca de dar clases en la escuela sobre la donación de órganos. A la mayoría de los niños se les permitió decidir por sí mismos a los 15-16 años.

Conclusión. Las conversaciones en familia sobre la donación son frecuentes entre los miembros de la pareja; sin embargo, es posible mejorar las charlas con los niños. Si se quisiera, podrían ofrecerse factores desencadenantes de estas conversaciones a través de la información pública y de la enseñanza, lo que también ayudaría a educar sobre esta cuestión de salud.

Palabras clave: niño, educación, relaciones familiares, donación de órganos, padres.
\end{abstract}

http:/ /dx.doi.org/10.5546/aap.2014.224

\section{INTRODUCCIÓN}

Pedir a los padres que tomen una decisión sobre la donación de órganos cuando acaban de informarles sobre la muerte de su hijo tal vez sea una de las situaciones más graves y difíciles de la vida. ${ }^{1}$ La decisión de donar los órganos está influenciada por muchos factores, como por ejemplo, los conocimientos sobre la donación, los valores culturales y religiosos, las circunstancias en que se produjo el fallecimiento, las experiencias hospitalarias y la edad del eventual donante. ${ }^{2,3}$

Además, también se deben tener en cuenta otros factores más personales: ¿los padres consideraron este asunto respecto de ellos mismos?, ¿lo hablaron entre sí o en un entorno familiar? En el caso de los adultos, la decisión real sobre la donación suele depender de si la persona responsable de tomar la decisión, en general un familiar, conoce o no los deseos del eventual donante. ${ }^{4}$

En el caso de un niño, la mayoría de las veces los familiares responsables de tomar la decisión son los padres. Incluso si los padres piensan que la donación de órganos es una cuestión importante, a menudo no han considerado la posibilidad de que su hijo se convierta en donante de órganos o tejidos. Es más, se sabe que raramente se habla sobre la donación con los niños en el entorno del hogar. ${ }^{5}$ Se desconoce si los padres hablan sobre esta cuestión entre sí o con sus hijos en un contexto no crítico. Sin embargo, tanto en la bibliografía como en nuestra práctica, se estipula que cuando los padres se ven confrontados de repente por la pregunta acerca de la donación y han hablado previamente 
sobre este tema con su hijo, tienen la necesidad de cumplir con los deseos del niño. ${ }^{3}$

En los Países Bajos, no se permite la donación de órganos por parte de niños menores de 16 años sin el consentimiento de los padres. En la bibliografía se halló que una conversación previa en familia constituye un sólido factor de predicción del consentimiento para donar. ${ }^{3,4,6-8}$ Waldrop y colaboradores indicaron que es necesario alentar a los familiares para que hablen sobre sus sentimientos respecto de la donación en circunstancias no críticas. ${ }^{3}$ En consecuencia, es importante fomentar las conversaciones en familia sobre la donación de órganos. Una manera de impulsar este tipo de conversaciones en familia es a través de la educación en la escuela. ${ }^{4}$

En general, según las pruebas empíricas, a partir de los 9 años los niños son cognitiva y emocionalmente capaces de brindar su asentimiento en condiciones normales. ${ }^{9}$ Además, las investigaciones han demostrado que, a partir de los 12 años, los niños son capaces de pensar sobre la donación de órganos, y están dispuestos a pensar en esta cuestión. ${ }^{5}$ Sin embargo, hasta donde sabemos, no existen estudios sobre los conocimientos de los padres respecto de la opinión de sus hijos menores de 12 años. La pregunta que surge es si los padres han considerado este asunto respecto de ellos mismos y han hablado sobre el tema entre sí o con sus hijos. Nuestra hipótesis es que los padres no suelen hablar de esta cuestión dentro de su entorno familiar.

Este estudio se diseñó para investigar si los padres y las familias han hablado sobre la donación de órganos con sus hijos y cuáles fueron los factores que influyeron dicha conversación, cuando la hubo. También solicitamos a los encuestados su opinión en relación con ofrecer clases en la escuela sobre la donación de órganos y cuál consideran que es el umbral de edad ideal para que un niño decida respecto de la donación.

\section{MATERIALES Y MÉTODOS}

Se reclutó a los encuestados de un panel en línea utilizado por la televisión pública, empresas comerciales y el gobierno. El objetivo de este panel es obtener opiniones sobre temas actuales y sociales. Las personas de este panel se seleccionan a través de avisos en diarios y revistas. Si bien el uso de Internet es una metodología válida para los estudios exploratorios, esta estrategia tiene ciertos riesgos de sesgo.

Se envió el cuestionario por correo electrónico a 14000 miembros del panel en noviembre de 2007. Se invitó a todos los miembros del panel a responderlo en forma anónima dentro de las cuatro semanas. Entre los encuestados, se seleccionó a las personas con hijos de 0 a 15 años de edad. Se eligieron estos límites de edad porque, según la ley de donación de órganos de los Países Bajos, las personas mayores de 16 años están autorizadas a decidir por sí mismas respecto de la donación de órganos. En el caso de los menores de 16 años, sus padres son sus representantes legales.

Las preguntas sobre la donación se integraron dentro de una encuesta más grande que incluía 88 puntos sociales diferentes actuales (por ejemplo, política, consumo mediático). Para este estudio, pudimos seleccionar los puntos relacionados con la donación de órganos, incluida la pregunta sobre la categoría de inscripción del encuestado en el registro de donantes de los Países Bajos: positiva (= consentida), negativa (= rechazada), neutra (decisión tomada por un familiar o una persona específica) o no inscripto.

En el Anexo 1 se incluyen las preguntas sobre la donación. Se extrajeron los grupos etarios de los padres (ver Tabla 1). También se extrajeron del conjunto de datos la religión y el tipo de educación de los padres (ver Tabla 2). Las religiones se dividieron en iglesia Católica Romana, otras religiones (incluidos el Islam y la iglesia protestante en los Países Bajos) y sin religión. El nivel educativo de los padres se dividió en alto (universidad, secundaria) y medio/bajo (primaria). Se seleccionaron dichas variables demográficas porque son factores de predicción conocidos de la actitud respecto de la donación de órganos..$^{5,10,11}$

Debido a la naturaleza del estudio, se realizó un análisis descriptivo. Los datos se analizaron con el software SPSS 17 (SPSS Inc., Chicago, IL, Estados Unidos). Las variables dependientes se estudiaron mediante la prueba del chi-cuadrado.

TABLA 1. Grupos etarios de los padres

\begin{tabular}{lc} 
Grupo etario & $\%$ \\
\hline 18-24 años & 0,8 \\
25-34 años & 6,9 \\
35-44 años & 57,3 \\
45-54 años & 32,5 \\
55-64 años & 2,3 \\
65+ años & 0,2 \\
\hline
\end{tabular}




\section{RESULTADOS}

Cuatro mil novecientos setenta y cinco $(35,5 \%)$ de los encuestados respondieron el cuestionario. Entre ellos, seleccionamos a las 1146 personas con hijos de 0 a 15 años de edad. De estos encuestados seleccionados, 1099 vivían en pareja. En total, 830 eran hombres y 316, mujeres. Los encuestados seleccionados estaban a cargo de 1591 niños distribuidos por edad: 478 padres tenían hijos de entre 0 y 5 años, 366 tenían hijos de entre 6 y 9 años, 258 tenían hijos de entre 10 y 11 años, y 489 tenían hijos de entre 12 y 15 años.

En la Tabla 2 se muestran las variables demográficas de religión, educación e inscripción como donante de los 1146 encuestados en comparación con la población general de los Países Bajos. ${ }^{12,13}$

Todos los encuestados respondieron que habían oído sobre la donación de órganos.

\section{Conversación en familia}

De los 1099 encuestados que vivían con una pareja, $925(84,2 \%)$ habían hablado sobre la donación de órganos con su pareja, mientras que $174(15,8 \%)$ nunca habían conversado sobre este tema. De los 1146 padres, 326 $(28,4 \%)$ habían conversado sobre este asunto con sus hijos (distribución por edad de los niños: entre 0 y 5 años, 5,9\%; entre 6 y 9 años, $23,1 \%$; entre 10 y 11 años, $41 \%$; y entre 12 y 15 años, 56\%). Si se combinan los datos para todos los niños de 10 a 15 años de edad, se obtiene un valor del $46,5 \%$. Dentro de la familia, son las madres quienes hablan sobre este tema con sus hijos más a menudo que los padres $(36,7 \%$ frente a $23,3 \%)$.

No se observó una relación entre el nivel educativo de los padres y el hecho de haber o no conversado sobre este tema entre ellos $\left(\chi^{2}\right.$, $p=0,426)$ o con sus hijos $\left(\chi^{2}, p=0,499\right)$.

No se halló una relación entre la religión y la prevalencia de las conversaciones entre los padres sobre la donación de órganos $\left(\chi^{2}, p=0,068\right)$, ni entre la religión y las conversaciones sobre este tema entre los padres y sus hijos $\left(\chi^{2}, p=0,793\right)$.

Los padres que se habían inscrito en el registro de donantes de los Países Bajos habían conversado sobre este tema con más frecuencia entre ellos $\left(\chi^{2}, p=0,018\right)$ y con sus hijos $\left(\chi^{2}\right.$, $p=0,014)$, en comparación con quienes no se habían inscrito. Asimismo, los padres inscritos en la categoría positiva incluso habían hablado con sus hijos con mayor frecuencia $(35,3 \%$ frente a $18,5 \%, \chi^{2}, p=0,01$ ) en comparación con los padres inscritos en la categoría neutra o negativa.

Entre los padres que habían conversado sobre la donación de órganos con sus hijos, los motivos indicados más frecuentemente fueron "es algo normal que un padre cumpla explícitamente con los deseos de sus hijos" y "es una cuestión importante"; a veces, los padres también indicaron que "es un tema de actualidad". En algunas ocasiones, los padres mencionaron que "fue un tema tratado en la escuela" y "mi hijo

TABla 2. Variables demográficas de los encuestados en comparación con la población general de los Países Bajos

\begin{tabular}{|c|c|c|c|}
\hline \multirow[t]{2}{*}{ Variables demográficas } & \multicolumn{2}{|c|}{$\begin{array}{c}\text { Encuestados } \\
n=1146 \\
830 \mathrm{H} / 316 \mathrm{M} \\
\end{array}$} & \multirow{2}{*}{$\begin{array}{c}\text { Población general } \\
\% \\
\end{array}$} \\
\hline & $\mathbf{N}$ & $\%$ & \\
\hline Religión & & & \\
\hline Católica Apostólica Romana & 255 & 22 & 29 \\
\hline $\begin{array}{l}\text { Otras religiones (incluidos la iglesia protestante } \\
\text { en los Países Bajos y el Islam) }\end{array}$ & 358 & 31 & 29 \\
\hline Sin religión & 533 & 47 & 42 \\
\hline Nivel educativo & & & \\
\hline Alto & 627 & 55 & 28 \\
\hline Medio/bajo & 519 & 45 & 72 \\
\hline Inscripción como donante & & & \\
\hline No inscrito & 517 & 45 & 59 \\
\hline Inscrito & 629 & 55 & 41 \\
\hline Categoría positiva (consentida) & 504 & 44 & 20 \\
\hline Categoría negativa (rechazada) & 65 & 6 & 11 \\
\hline $\begin{array}{l}\text { Categoría neutra (decisión tomada por } \\
\text { un familiar o una persona específica) }\end{array}$ & 60 & 5 & 10 \\
\hline
\end{tabular}


quiso conversar sobre el tema desencadenado a partir de los medios (sociales)".

En el caso de los padres que no deseaban hablar sobre este tema, los motivos más frecuentes fueron "creo que mi hijo es muy pequeño aún" y "nunca pensé en hablar sobre este tema con mi hijo". A veces, los padres también indicaron "no quiero pensar en este tema". Unas pocas veces, el motivo fue "no quiero ser donante" o "no tengo tiempo para hablar sobre esto".

\section{Clases escolares}

De los 1146 padres, 796 (69,4\%) estuvieron a favor de ofrecer clases sobre este tema en la escuela, $166(14,5 \%)$ demostraron una actitud neutra y $184(16,1 \%)$ estuvieron en contra de las clases escolares.

En la Figura 1 se muestra el umbral de edad para estas clases. De los 962 padres con una opinión positiva o neutra, el $60,7 \%$ consideran que el mejor momento para iniciar estas clases es en la escuela primaria (es decir, a los 10-12 años o antes de esta edad).

No se halló una relación entre la opinión de los padres acerca de las clases en la escuela y su nivel educativo $\left(\chi^{2}, p=0,697\right)$. Además, tampoco se observó una relación entre la religión y la opinión de los padres respecto de las clases en la escuela $\left(\chi^{2}, p=0,108\right)$.

La inscripción de los padres en el registro de donantes de los Países Bajos mostró una relación significativa con la opinión sobre las clases $\left(\chi^{2}, p<0,001\right)$. Los padres inscritos en la categoría positiva más a menudo consideran que las clases en la escuela sobre la donación de órganos son una herramienta adecuada para enseñar a los niños, en comparación con los padres con categoría de inscripción negativa $\left(85,1 \%\right.$ frente a $\left.46,2 \%, \chi^{2}, p<0,001\right)$. Asimismo, los padres inscritos en la categoría neutra con mayor frecuencia tienen una actitud positiva respecto de la enseñanza, en comparación con los padres inscritos en la categoría negativa $(73,3 \%$ frente a $46,2 \%)$.

La prevalencia de la conversación sobre este tema por parte de los padres no estuvo relacionada con las ideas de los padres respecto de las clases en la escuela. Sin embargo, los padres que habían hablado sobre la donación de órganos con sus hijos tenían una actitud significativamente más positiva respecto de las clases $\left(\chi^{2}, p<0,001\right)$.

\section{Umbral de edad ideal para decidir}

Se preguntó a los encuestados a qué edad

Figura 1. Porcentaje y cantidad de encuestados (eje vertical) frente al umbral de edad ideal para recibir una clase en la escuela sobre la donación de órganos (eje horizontal)

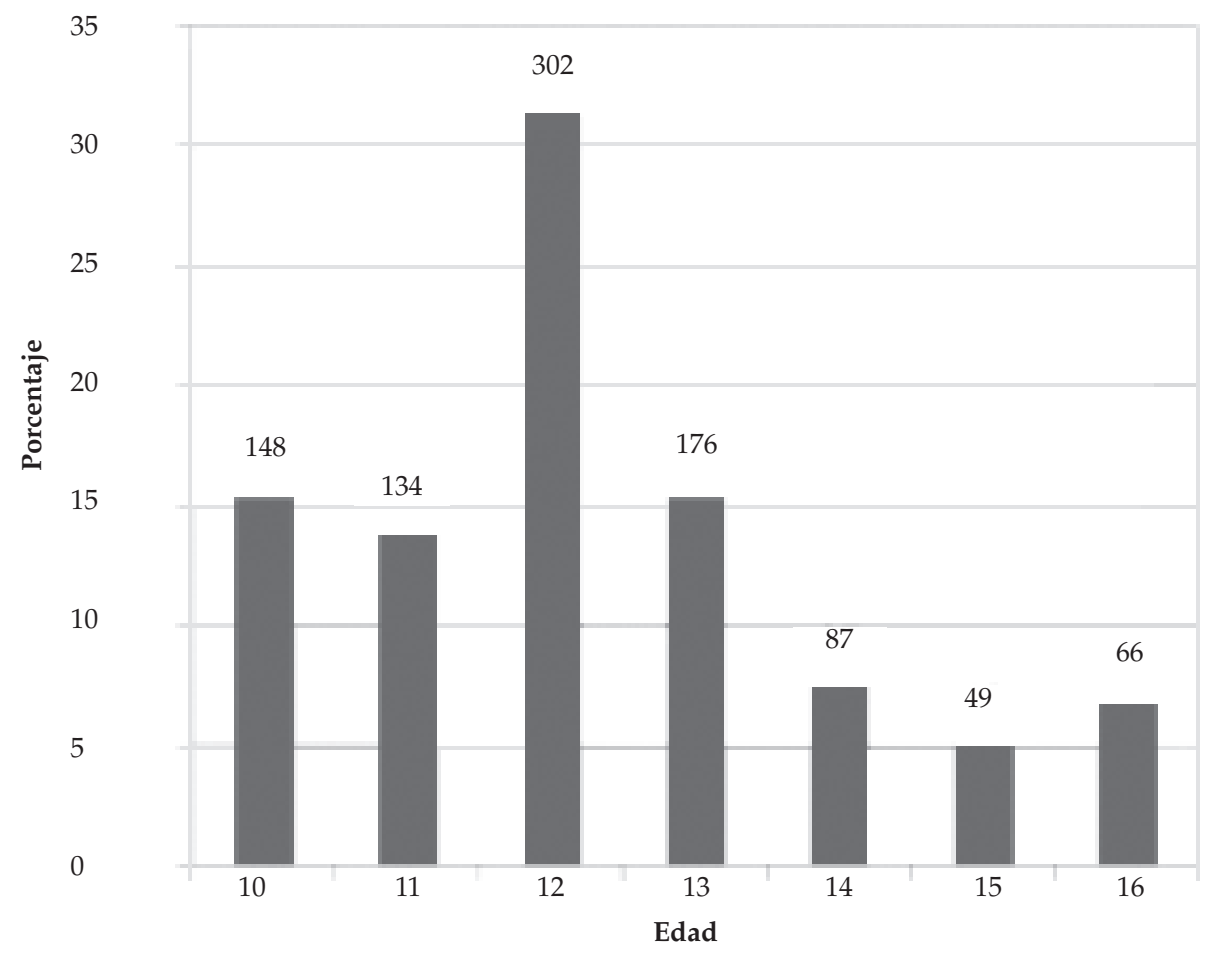


deberían permitir que sus hijos decidan por primera vez acerca de convertirse en donantes (ver Tabla 3).

Se halló una relación entre la opinión de los padres y lo que ellos consideraban que era la edad ideal para decidir; los padres sin religión o pertenecientes a la iglesia Católica Romana creían que sus hijos deberían decidir a una edad más temprana que los padres de otra religión $\left(\chi^{2}\right.$, $p<0,01$ ).

El nivel educativo de los padres también estuvo relacionado con lo que los padres consideraban que era la edad ideal para decidir sobre ser donantes. Los padres con un nivel educativo más alto suelen pensar que sus hijos son capaces de tomar una decisión a una edad más temprana que los padres con un menor nivel educativo $\left(\chi^{2}, p=0,04\right)$.

Además, también hubo una relación con la inscripción de los padres en el registro de donantes de los Países Bajos. De los padres inscritos, el 50\% consideraba que sus hijos deberían ser capaces de decidir antes de los 15 años; en el caso de los padres que no estaban inscritos, este valor fue del $20 \%\left(\chi^{2}, p<0,01\right)$.

\section{DISCUSIÓN}

En este estudio, exploramos las opiniones de los padres de los Países Bajos respecto de la donación de órganos por parte de sus hijos y el alcance de las conversaciones sobre este tema en el entorno familiar. Hasta donde sabemos, este es el primer estudio sobre las conversaciones en familia entre los padres, y entre los padres y los niños, acerca de la donación de órganos.

Este estudio muestra que, en los Países Bajos, la mayoría de los padres (84\%) han conversado con su pareja sobre este tema. En promedio, el $46,5 \%$ de los padres han hablado de este tema con sus hijos de entre 10 y 15 años. En general, son las madres quienes hablan de este asunto más a menudo que los padres. De todos los padres, la mayoría (56\%) comenzaron a conversar del tema cuando sus hijos tenían 12 años. Este último valor

TABLA 3. Umbral de edad ideal para tomar la decisión de ser donante

\begin{tabular}{lcc}
\hline Edad & $\mathbf{N}$ & $\mathbf{\%}$ \\
\hline $9 / 10$ años & 84 & 7,3 \\
$11 / 12$ años & 112 & 9,8 \\
$13 / 14$ años & 149 & 13,0 \\
$15 / 16$ años & 801 & 69,9 \\
\hline
\end{tabular}

corresponde a los hallazgos informados en los niños de un estudio previo acerca de las opiniones de los niños sobre la donación de órganos. Aquel estudio, que tenía un diseño similar, demostró que el $43 \%$ de los niños de entre 12 y 15 años informaron que habían conversado sobre este tema en su casa más de una vez. ${ }^{5}$ En nuestro estudio, el alcance de las conversaciones en familia no estuvo relacionado con el nivel educativo o la religión de los padres. Además, no es sorprendente que los padres inscritos en el registro de donantes de los Países Bajos conversaran sobre este tema con sus familias con mayor frecuencia. Esto concuerda con los hallazgos de Morgan y Miller en adultos; quienes habían firmado su tarjeta de donante, la mayoría habían expresado sus deseos a sus familiares. ${ }^{14}$

En nuestro estudio, los padres también indicaron distintos motivos para no iniciar una conversación sobre este tema. Uno de los motivos fue que nunca habían pensado sobre la posibilidad de que sus hijos fueran donantes. Esto no es extraordinario debido a que las campañas de información pública holandesas sobre la donación de órganos se centran solamente en la donación por parte de los adultos; los padres no son conscientes del hecho de que también es factible la donación por parte de los niños. Es posible imaginar que la conciencia de los padres respecto de la donación de órganos es mayor a medida que sus hijos crecen. Un segundo motivo indicado por los padres para evitar la conversación en familia fue que no querían pensar en un tema tan complejo.

Los padres que comenzaron a hablar del tema con sus hijos creían que se trataba de una tarea que les correspondía como padres. Se halló que, con frecuencia, la atención de los medios (por ejemplo, el tema tratado en un programa de noticias para niños, campañas públicas) fue un desencadenante para comenzar esta conversación. Cuando fueron los niños quienes iniciaron la conversación, fue también a veces desencadenado por los medios sociales. En los Países Bajos, casi todos los niños utilizan activamente los medios sociales, y estos medios confrontan a los niños cada vez más con diversos temas sociales. ${ }^{15}$ Otro motivo mencionado por los padres es que los niños comienzan la conversación en el hogar a partir de una clase en la escuela. Consideramos que pueden usarse estos canales de comunicación, como los medios sociales y las clases en la escuela, para salvar la diferencia existente entre los padres y los niños sobre este tema. 
La mayoría de los padres respaldan la idea de ofrecer clases en la escuela sobre la donación de órganos. Los padres inscritos en la categoría positiva en el registro de donantes fueron quienes más apoyo demostraron. Cantarovich y colaboradores indicaron que, después de recibir educación en la escuela sobre la donación de órganos, los niños esperaban que sus familias se interesaran en la donación de órganos. ${ }^{16}$ Siminoff y colaboradores mencionaron que la educación es el mejor mecanismo para informar al público y preparar a las familias para el momento en que se les solicite la donación de órganos. ${ }^{4}$ Además, Randhawa señaló que los programas escolares pueden fomentar las conversaciones activas sobre la donación de órganos al tratarla como un asunto que contribuye al desarrollo social de los niños. ${ }^{17}$

También preguntamos a los padres cuál consideraban la edad ideal para que un niño decida convertirse en donante. Si bien la mayoría de los padres $(70 \%)$ creían que la mejor edad para que los niños decidan por sí mismos eran los 15-16 años, el 30\% de los padres pensaron que sus hijos eran capaces de decidir a una edad más temprana. Se halló una relación entre la religión de los padres y la edad en que los niños deberían ser capaces de decidir por sí mismos respecto de la donación de órganos. Los padres no religiosos o de la iglesia Católica Romana consideraban que sus hijos eran capaces de decidir a una edad más temprana que los padres pertenecientes a otras religiones. Sin embargo, no existen otros estudios con los cuales comparar estos resultados. Cabe destacar que se halló una relación entre el nivel educativo de los padres y la edad ideal para decidir; los padres con un nivel educativo alto consideraban que sus hijos eran capaces de decidir a una edad más temprana. En cambio, estos padres no hablaban más frecuentemente sobre la donación de órganos con sus hijos. Es necesario que se realicen estudios futuros para analizar estos resultados en perspectiva.

Se deben destacar algunas limitaciones. Este estudio se diseñó para investigar si los padres y las familias hablaban sobre la donación de órganos y estudiar los factores que influyen en la conversación. Dado que fuimos los primeros en hacerlo, optamos por un estudio descriptivo y desconocíamos los efectos o las diferencias de grupo previsibles, como por ejemplo las diferencias de género. Si bien la muestra era grande, los resultados de los cuestionarios autoadministrados y las diferencias de grupo deben interpretarse con precaución debido a una posible falta de potencia estadística. Aunque los datos se obtuvieron en 2007, consideramos que los hallazgos aún tienen vigencia. Raramente se habla sobre la donación de órganos de niños en los Países Bajos y, en consecuencia, la situación actual no debe haber diferido mucho en comparación con el período del estudio. A pesar de que hubo un exceso de representación de encuestados hombres, especulamos que nuestros datos son igualmente generalizables. Se prevé que exista cierto sesgo dado que el nivel educativo de los encuestados es levemente más alto que el estándar de la población holandesa. Asimismo, si bien las preguntas relacionadas con la donación estaban dentro de un cuestionario más amplio y el porcentaje de inscripción en el registro de donantes de los Países Bajos era similar a la población holandesa, existió cierto sesgo; el porcentaje de los encuestados inscriptos en la categoría positiva fue mayor en comparación con la generalidad de la población de los Países Bajos.

A pesar de estas limitaciones, deseamos destacar que emprendimos este estudio con el objetivo de identificar el alcance de las conversaciones entre padres y sus familias acerca de la donación de órganos por parte de los niños. Es sabido que las conversaciones en familia constituyen un factor clave para una adecuada toma de decisiones respecto de la donación de órganos. A partir de este estudio, sabemos que hay un margen para mejorar en términos de la conversación dentro del entorno familiar. Los medios y la atención de los medios sociales y la educación a nivel escolar pueden emplearse como desencadenantes. Los padres respaldan el desarrollo de estos programas educativos destinados a los niños y sus padres. Asimismo, las campañas públicas también deben apuntar a los adultos en su papel de padres y enfocarse además en las conversaciones en familia para ofrecer a los niños y sus padres oportunidades adecuadas para recibir la información correspondiente.

\section{CONCLUSIÓN}

\section{Puntos clave}

- La mayoría de los padres nunca hablaron sobre la donación de órganos con sus hijos.

- Los padres respaldan que se dicten clases sobre donación en la escuela.

- La mayoría de los padres consideran que la edad ideal para decidir convertirse en donante es a los 15 años. 
- En relación con las políticas de salud: es necesario fomentar las conversaciones en familia entre los padres y los niños acerca de la donación.

\section{Agradecimientos}

Agradecemos a No Ties por su participación en la evaluación de los datos y su predisposición a compartir sus datos con fines de investigación. También agradecemos a Rosanne van der Krol (estudiante de la maestría de psicología) y a la Dra. Karin Vermeulen (investigadora de evaluación de la tecnología sanitaria) por sus contribuciones.

\section{BIBLIOGRAFÍA}

1. Bellali T, Papadatou D. Parental grief following the brain death of a child: does consent or refusal to organ donation affect their grief? Death Stud 2006;30(10):883-917.

2. Siebelink MJ, Albers MJ, Roodbol PF, van de Wiel HB. Key factors in paediatric organ and tissue donation: an overview of literature in a chronological working model. Transpl Int 2012;25(3):265-71.

3. Waldrop DP, Tamburlin JA, Thompson SJ, Simon M. Life and death decisions: using school-based health education to facilitate family discussion about organ and tissue donation. Death Stud 2004;28(7):643-57.

4. Siminoff LA, Gordon N, Hewlett J, Arnold RM. Factors influencing families' consent for donation of solid organs for transplantation. JAMA 2001;286(1):71-7.

5. Siebelink MJ, Geerts EA, Albers MJ, Roodbol PF, van de Wiel HB. Children's opinions about organ donation: a first step to assent? Eur J Public Health 2012;22(4):529-33.

6. Walker JA, McGrath PJ, MacDonald NE, Wells G, et al. Parental attitudes toward pediatric organ donation: a survey. CMAJ 1990;142(12):1383-7.
7. Rodrigue JR, Cornell DL, Howard RJ. Pediatric organ donation: What factors most influence parents' donation decisions? Pediatr Crit Care Med 2008;9(2):180-5.

8. Oliver RC, Sturtevant JP, Scheetz JP, Fallat ME. Beneficial effects of a hospital bereavement intervention program after traumatic childhood death. J Trauma 2001;50(3):440-6.

9. Ondrusek N, Abramovitch R, Pencharz P, Koren G. Empirical examination of the ability of children to consent to clinical research. J Med Ethics 1998;24(3):158-65.

10. Reubsaet A, BrugJ, van den Borne B, van Hoof H. Predictors of organ donation registration among Dutch adolescents. Transplantation 2001;72(1):51-6.

11. Smits M, van den Borne B, Dijker AJ, Ryckman R. Increasing Dutch adolescents' willingness to register their organ donation preference: the effectiveness of an education programme delivered by kidney transplantation patients. Eur J Public Health 2006;16(1):106-10.

12. Siebelink MJ. (Comunicación personal). Dutch Donor Register; figures on registration 2007. 2009-2012.

13. Centraal Bureau voor de Statistiek, Statistics Netherlands. Statline. 2007. Disponible en: http:/ / www.cbs.nl/en-GB / menu/themas/urije-tijd-cultuur/publicaties/artikelen/ archief/2008/2008-2476-wm.htm y http://www.cbs.nl/ en-GB/menu/themas/onterwijs/publicaties/artikelen/ archief/2013/2013-3905-wm.htm [Consulta: 21 de febero de 2014].

14. Morgan SE, Miller JK. Beyond the organ donor card: the effect of knowledge, attitudes, and values on willingness to communicate about organ donation to family members. Health Commun 2002;14(1):121-34.

15. van Rooij AJ, Schoenmakers TM. Factsheet social media en gamen. Disponibleen:http:/ / www.ivo.nl/UserFiles/File/ Publicaties/2013-01\%20Factsheet\%20Social\%20media\%20 en\%20gamen.pdf. [Consulta: 22 de febrero de 2013].

16. Cantarovich F, Fagundes E, Biolcalti D, Bacqué MC. School education, a basis for positive attitudes toward organ donation. Transplant Proc 2000;32(1):55-6.

17. Randhawa G. Targeting the UK's young potential donors: placing organ donation on the school agenda. Transplantationsmedizin 1998;10:38-40. 


\section{Anexo 1}

El cuestionario contenía las siguientes preguntas (traducción del cuestionario original):

1. ¿Alguna vez oyó acerca de la donación de órganos o tejidos?

Sí / No

2. ¿Alguna vez habló sobre la donación de órganos con su pareja?

Sí / No

3. ¿Cuál fue el motivo por el cual habló de este tema con su pareja?

Respuesta abierta

4. ¿Por qué nunca habló de este tema con su pareja?

Respuesta abierta

5. ¿Alguna vez habló sobre la donación de órganos con su hijo?

Sí / No

6. ¿Cuál fue el motivo por el cual habló de este tema con su hijo?

Respuesta abierta

7. ¿Qué edad tenía su hijo cuando hablaron de este tema?

Grupos etarios

8. ¿Por qué nunca habló de este tema con su hijo?

Respuesta abierta

9. ¿Cuál es su opinión sobre las clases en la escuela acerca de la donación de órganos?

Escala de 5 puntos

10. ¿Cuál es la edad ideal para comenzar con este tipo de clases?

Grupos etarios

11. ¿A qué edad debería su hijo tomar su propia decisión respecto de la donación? Grupos etarios 